\title{
Detection of ATP-Binding to Growth Factors
}

\author{
Simone König, ${ }^{a}$ Anja Hasche, ${ }^{\mathrm{b}}$ Stefanie Pallast, ${ }^{\mathrm{b}}$ Josef Krieglstein, ${ }^{\mathrm{b}}$ and \\ Susanne Klumpp ${ }^{\mathrm{b}}$ \\ a Integrated Functional Genomics, Interdisciplinary Center of Clinical Research, University of Münster, \\ Münster, Germany \\ ${ }^{b}$ Institute for Pharmaceutical and Medicinal Chemistry, University of Münster, Münster, Germany
}

It was shown in previous work that the interaction of growth factors (GFs) with adenosine triphosphate (ATP) is essential for their neuroprotective effect. Here we investigated the nature of the association of human basic fibroblast growth factor (bFGF), nerve growth factor (NGF), and brain-derived neurotrophic factor (BDNF) with ATP. It was demonstrated that this interaction involves the formation of non-covalent ATP-GF complexes that are labile at low $\mathrm{pH}$ and that could be selectively purified and subjected to electrospray and MALDI-TOF mass spectrometry. The results obtained with these techniques indicated that the stability of the complexes is high. Main features of the procedure used here are: (1) reversed-phase purification of nucleotide-protein non-covalent complexes, (2) their detection with MALDITOF-MS using acid-free matrix, and/or (3) their measurement with ESI-MS using soft desolvation conditions. The methodology was successful in providing proof for the presence of various nucleotide-GF complexes. It was extended to other nucleotide-binding proteins (ribonuclease A) as well as proteins that do not exhibit nucleotide binding (lysozyme) as positive and negative control, respectively. Thus, the method demonstrated its general use for the investigation of a wide range of proteins interacting with nucleotides as long as their complexes are sufficiently stable to accommodate the experimental conditions. (J Am Soc Mass Spectrom 2008, 19, 91-95) (c) 2008 American Society for Mass Spectrometry

$\mathrm{T}$ The interaction of growth factors (GFs) such as human basic fibroblast growth factor (bFGF, $17,052 \mathrm{Da})$, nerve growth factor (NGF, 13,261 Da), and brain-derived neurotrophic factor (BDNF, 13,506 Da) with adenosine triphosphate (ATP) has only recently been discovered and it has been shown to be essential for their neuroprotective effect [1]. Radioactive labeling of the GFs with $\left[\gamma^{-32} \mathrm{P}\right]$ ATP was destroyed at low $\mathrm{pH}$, but was stable in basic buffers. The association of ATP with bFGF was still detectable after denaturing gel electrophoresis. Because no autokinase activity of the GFs was known, covalent phosphorylation did not seem likely. Nevertheless, both covalent and non-covalent interactions were investigated by using electrospray ionization (ESI) and matrix-assisted laser/desorption ionization time-of-flight mass spectrometry (MALDI-TOF-MS).

MS-detection of $\mathrm{N}$ - or acylphosphates is not far advanced due to the difficulties associated with the acid lability of the modification [2-6]. The investigation of non-covalent binding in the gas phase is also not trivial because complexes that are stable in liquid medium may not be observed easily in the gas phase [7]. Even when signals are detected the question remains to what

Address reprint requests to Dr. Simone König, University of Münster, Integrated Functional Genomics, Interdisciplinary Center of Clinical Research, Röntgenstr. 21, 48149 Münster, Germany. E-mail: koenigs@unimuenster.de extent gas-phase ions reflect biological conditions. In the case of nucleotide binding to proteins it needs to be considered that nucleotides themselves are acid-labile and thus demand moderate assay conditions. Only few studies have documented the use of MS for the analysis of protein-nucleotide complexes [8-11]. The authors of these studies all electrospray the complexes from a solution containing the necessary components (cations, nucleotide, protein) and a volatile buffer imitating physiological conditions. However, both in MALDI and ESI unspecific gas-phase adduct formation needs to be considered to different degrees. Attempts to avoid adducts involve harsher desolvation conditions (i.e., high temperature) and they put strain on non-covalent complexes as well. Therefore, the measurement of protein complexes with MS requires a sufficiently stable bond as well as a delicate balance of the experimental parameters. Here, we present a method that allows the preservation of nucleotide-protein complexes and their detection with soft ionization MS [12].

\section{Experimental}

Chemicals were purchased from Sigma-Aldrich (Seelze, Germany) unless otherwise noted. Commercial NGF and BDNF for comparative experiments were obtained from Acris (Hiddenhausen, Germany). The plasmids pET11a-proNGF or pET11a-proBDNF were a 
kind gift from E. Schwarz (University of Halle, Germany) and P. Madsen (University of Aarhus, Denmark), respectively.

Recombinant human GFs were expressed in Escherichia coli. bFGF was expressed and purified as previously described after deletion of the codon for the second amino acid (alanine) in the sequence of the bFGF gene [1]. Expression of NGF and BDNF in the presence of their covalently attached pro-domain in E. coli Rosetta(DE3)pLysS carrying the plasmids pET11aproNGF or pET11a-proBDNF, respectively, led to the formation of inclusion bodies. After their solubilization, proNGF and proBDNF were refolded and purified as described by Rattenholl and colleagues [13] (except that for BDNF no hydrophobic interaction chromatography was performed) with subsequent cleavage of the propeptides by trypsin. Labeling of GF proteins ( $5 \mu \mathrm{g}$ bFGF or $3.5 \mu \mathrm{g}$ NGF or BDNF) was carried out at $37^{\circ} \mathrm{C}$ for 15 and $30 \mathrm{~min}$, respectively, in a volume of $15 \mu \mathrm{L}$ containing $25 \mathrm{mM}$ Tris $/ \mathrm{HCl}(\mathrm{pH} 7.5)$ and $0.1 \mathrm{mM}$ ATP. For labeling of bFGF $0.1 \mathrm{mM} \mathrm{MgCl} 2$ and $1 \mathrm{mM}$ DTT were added, whereas NGF and BDNF were incubated with $0.1 \mathrm{mM} \mathrm{MnCl}{ }_{2}$. Ribonuclease A from bovine pancreas $(7.5 \mu \mathrm{g})$ and lysozyme from chicken egg white $(3.5 \mu \mathrm{g})$ were treated with ATP in the same way as described for GFs, except that no cations were added for labeling of ribonuclease $\mathrm{A}$.

For purification of the protein and its complex, ZipTips $\mathrm{C}_{4}$ (Millipore, Bedford, MA, USA) were washed with elution buffer $(80 \%$ methanol, $0.1 \%$ acetic acid) and equilibrated using aqueous solvent (5\% methanol, $0.1 \%$ acetic acid). For sample binding, $10 \mu \mathrm{L}$ of solutions of GFs $(3 \mathrm{mg} / \mathrm{mL})$ or control proteins (3 $\mathrm{mg} / \mathrm{mL}$ ) were applied and rinsed with equilibration solution. The purified complexes of NGF and BDNF with ATP were eluted in $5 \mu \mathrm{L}$ and bFGF-ATP in $10 \mu \mathrm{L}$ elution solvent.

For MALDI-MS MALDImicroMX (Waters Corp., Milford, MA, USA) was used in positive linear mode. After preparation of the target with $0.5 \mu \mathrm{L}$ of $10 \mathrm{mg} / \mathrm{mL}$ sinapinic acid in acetone, $0.5 \mu \mathrm{L}$ of each sample, and 10 $\mathrm{mg} / \mathrm{mL}$ sinapinic acid in $40 \%$ acetonitrile were added. For partial proteolysis of NGF trypsin was used in a ratio of 1:40. Samples were analyzed by MS after $0,1,2$, $5,7,10$, and $30 \mathrm{~min}$ of incubation at $37^{\circ} \mathrm{C}$. ESI-MS was performed with Esquire ${ }_{3000}$ (Bruker Daltonics, Bremen, Germany) using a modified manual liquid-junction nanospray source with self-pulled capillaries [14]. Drying gas was maintained at $100{ }^{\circ} \mathrm{C}$. As mentioned in the text either normal or extended mass range was used applying a trap target mass as given later in Figure 3.

\section{Results and Discussion}

We adjusted all protocols to accommodate the acidlabile character of the protein modification. So far, there was no mass spectrometric evidence for the covalent attachment of phosphate groups to GFs. However, our results indicated the formation of ATP-GF non-covalent

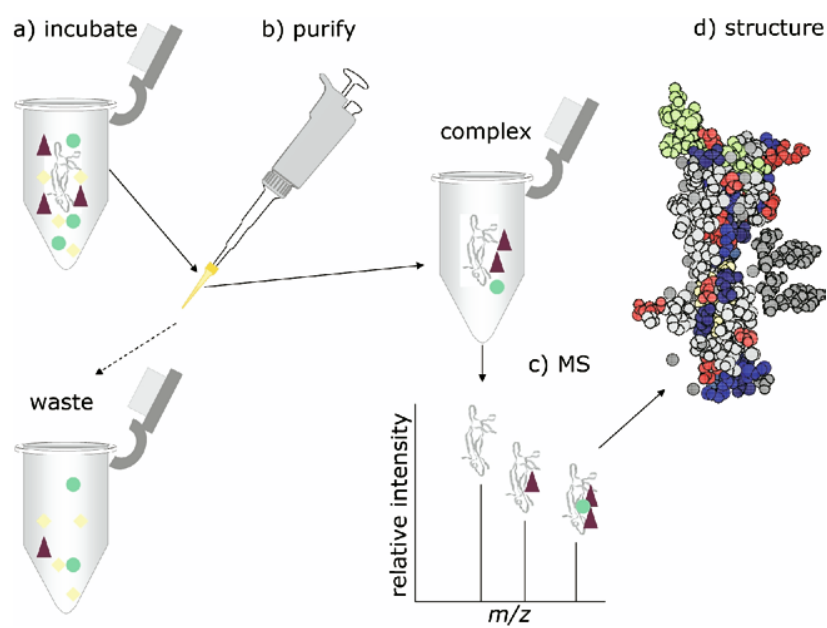

Figure 1. Scheme of the workflow for the detection of noncovalent complexes of GF proteins with nucleotides. The protein image of NGF was modeled using PDB entry 1BET. Triangles symbolize ATP, circles the divalent cation, and squares buffer ions. (a) GF was incubated with ATP and $\mathrm{Mg}^{2+}$ or $\mathrm{Mn}^{2+}$. (b) Salts and unbound material were removed using reversed-phase material. (c) The purified complex was measured in MALDI-TOF and ESI-MS. (d) The procedures were applied to structural investigations such as time-dependent proteolysis. The structure was visualized using PDB entry 1BET and CS Chem3D Pro 3.5.1. Two molecules of ATP were added for size comparison.

complexes. Methods for the investigation of non-covalent binding of proteins using MS require optimization concerning buffers and experimental parameters. Physiological buffers that are needed to preserve the complex tend to suppress the analyte signal. Usually, volatile buffers such as ammonium bicarbonate or acetate are used as a compromise. This approach was not successful here. However, derived from the efforts of providing high-pH conditions parameters were found that allowed the detection of ATP-GF complexes. For the removal of residual contaminants in GF samples dialysis and solid-phase extraction worked best. In fact, desalting using $C_{4}$ material allowed us to purify the ATP-GF complexes when harsh solvents were avoided. Methanol was used instead of acetonitrile and a weak acid (formic or acetic) was added. The latter turned out to be crucial; otherwise, MS signals were noisy and unresolved. Labeling of GFs with ATP was resistant to low-pH treatment during this procedure as could be demonstrated using autoradiography. The flowchart of the desalting procedure is depicted in Figure 1.

Although well-resolved spectra of intact NGF (see Figure $3 a$ ), bFGF and BDNF could be generated with an ion trap, only MALDI-TOF-MS allowed detection of the purified ATP complexes at first. As shown in Figure 2 the attachment of one and two nucleotide molecules and a divalent cation to NGF and bFGF was observed. Unspecific adduct formation could be excluded because excess unbound ATP and cations were removed by the purification step. Protein-free control experiments revealed that only $1 \%$ of unbound $\left[\gamma^{32} \mathrm{P}\right] \mathrm{ATP}$ was transferred to the eluate. However, breakdown of complex 


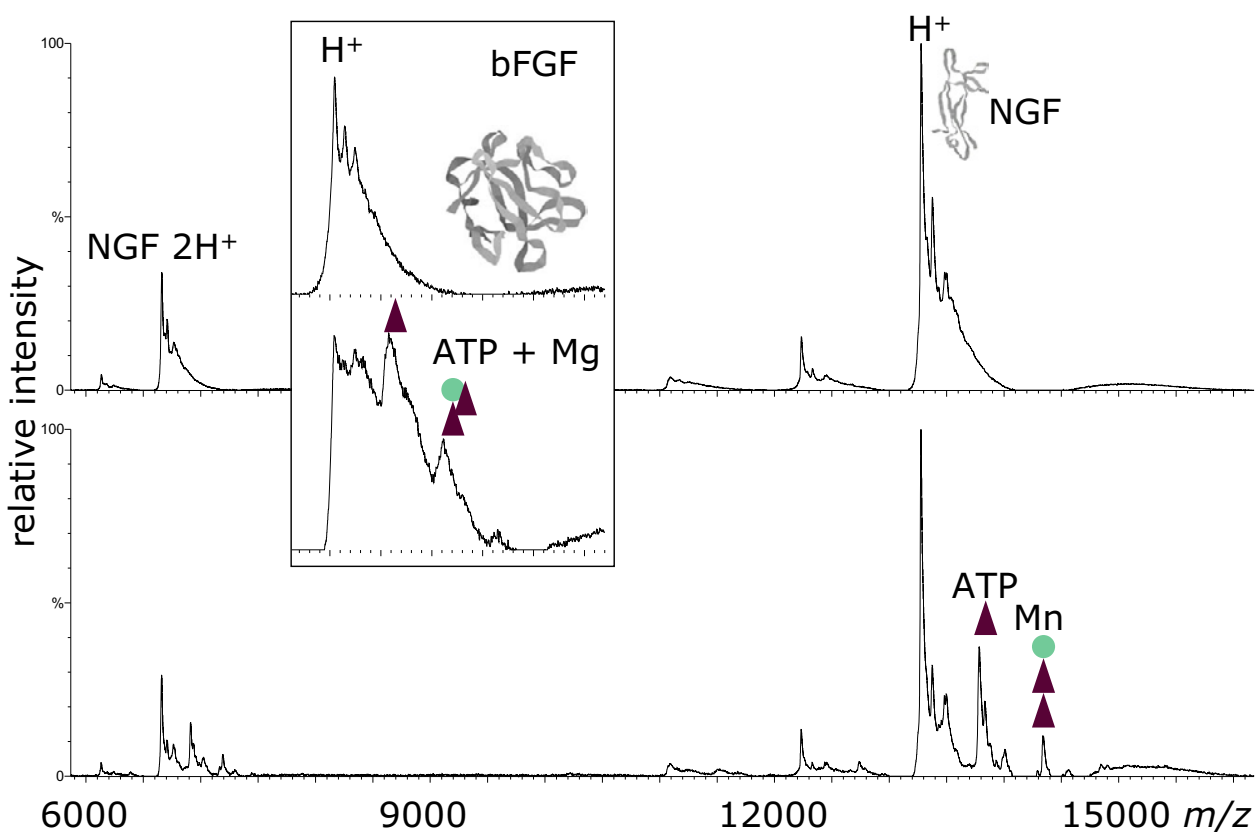

Figure 2. MALDI-TOF spectrum of purified NGF-ATP-Mn complexes (singly and doubly charged ions and low abundant cleavage products at $\sim 12 \mathrm{kDa}$ ) and bFGF-ATP-Mg complexes (inset) measured with acid-free sinapinic acid matrix. The structure for bFGF was modeled using 1BAS. One and two molecules of ATP $(507.2 \mathrm{~g} / \mathrm{mol})$ and one corresponding cation $\mathrm{Mg}^{2+}(24.3 \mathrm{~g} / \mathrm{mol})$ or $\mathrm{Mn}^{2+}(54.9$ $\mathrm{g} / \mathrm{mol}$ ) were complexed and appear as additional peaks as annotated with triangles and circles. For further annotations see Figure 1.

ions during ionization had to be considered and could have been partially responsible for the GF ions containing one or no ATP molecule. Complexation of two molecules of ATP assisted by the binding of one cation appeared to be the natural process that confirmed previously published data showing the labeling of bFGF, NGF, and BDNF with $\left[\gamma_{-}^{-32}\right.$ P]ATP and the interaction of bFGF with $\left[\alpha^{-}{ }^{32} \mathrm{P}\right]$ ATP [1]. The stability of the complexes appeared to be high because they survived the purification procedure.

The MALDI matrix had to be acid free, however. Although the addition of TFA to the MALDI matrix proved to be important for preventing solvent adducts to the protein peaks, it also eliminated any signal from the non-covalent complex. Therefore, the observed adducts of $98 \mathrm{Da}$ (Figure 2) could not be easily avoided. These satellite peaks were present in all GF samples tested, including commercial products independent of treatment with ATP. Labeling of GFs with radioactive ortho-phosphate was not detectable. Although the presence of phosphoric acid or sulfate is suggested by the mass difference, so far the nature of these adducts remains ambiguous to us and to other authors $[8,15$, 16].

Different MS techniques can often provide complementary information and, in this case, the resolution of protein charge states was expected using ESI-MS. The Esquire $_{3000}$ ion trap was available for that purpose. Although this analyzer type is not commonly used for the measurement of natural complexes, it has been shown that it is possible to detect multimers of bovine serum albumin and alcohol dehydrogenase with the commercial instrument [17]. More specific experiments such as the collision-induced dissociation measurements of trypsin/trypsin inhibitor complexes were performed on modified instrumental configurations [15]. Soft desolvation conditions were applied to preserve the complex of ATP with GFs. As depicted in Figure 3a for NGF, the use of the trap's different measurement ranges allowed us to observe several ion populations. Signals could be detected for unfolded and partially folded NGF monomers as well as dimers, the naturally occurring state of NGF [18]. For bFGF and BDNF no multimers were detected. However, the addition of ATP caused the peaks of the higher mass/lower charge ions to broaden with very little resolution of the individual molecular species in the case of bFGF and NGF (data not shown). Nevertheless, the BDNF-ATP complex could be observed with resolved charge-state envelopes of all complex ion species (Figure $3 b$ ).

Although the protocol was applied to bFGF, NGF, and BDNF in the same way, the proteins differed in their behavior and that was likely associated with structural properties. Purification of bFGF seemed to be less successful than that of NGF and BDNF, possibly due to its spherical structure [19] (Protein Databank PDB entry 1BAS) hindering salt diffusion. In contrast, NGF (1BET) and BDNF (1BND) folding is determined by three disulfide bridges and an elongated $\beta$-sheet structure [20]. The stability of the various complexes had to be tested individually because some survive ionization better than others. 

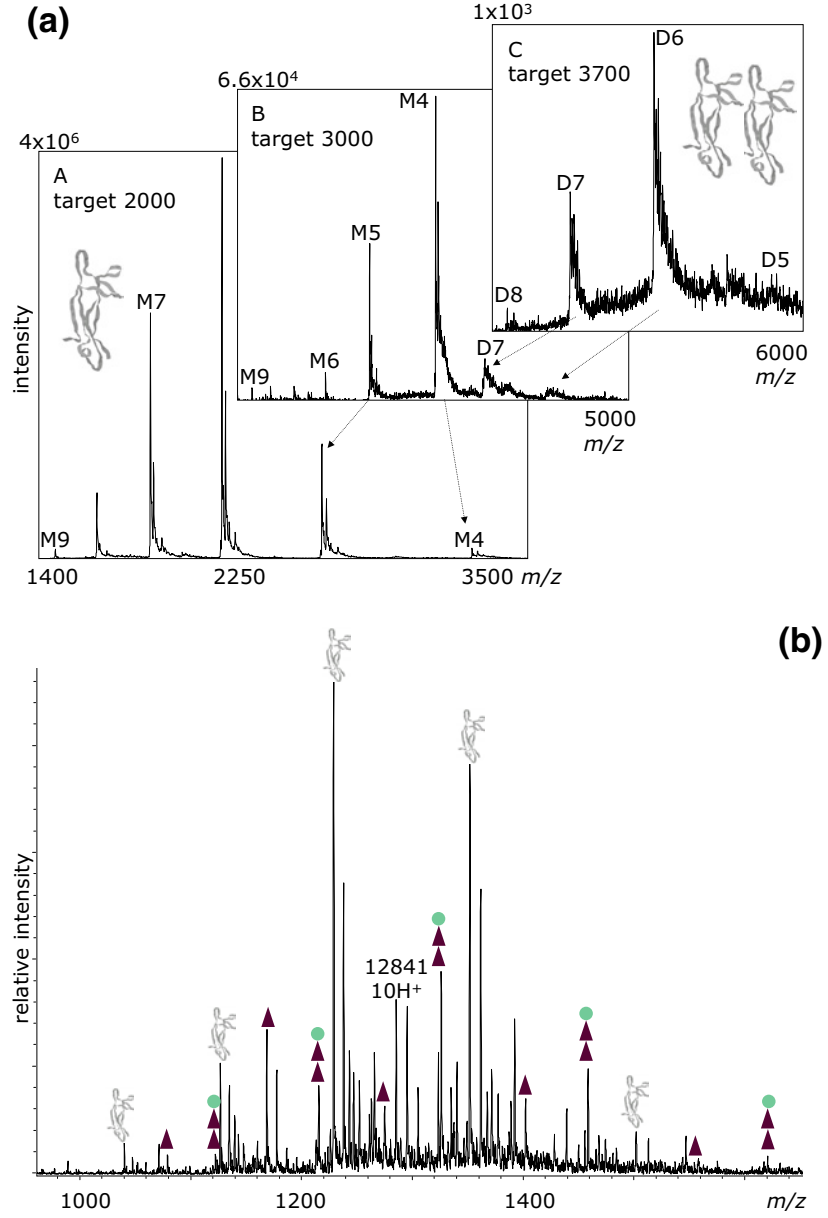

Figure 3. (a) Ion trap data of purified native NGF. Charge state envelopes were observed for the monomer at trap target mass 2000 accompanied by residual $98 \mathrm{Da}$ satellite peaks (see text). Changing analyzer parameters brings up ions for the NGF dimer (center spectrum), which can be further enhanced monitoring the ion population in the high mass range (top right). (b) Ion trap spectrum of purified BDNF-ATP complex at trap target mass 900 . The spectrum is complex due to the presence of a second smaller protein, which supposedly is a cleavage product of BDNF derived from the tryptic removal of the pro-peptide during the production process of the GF. So far, it could not be removed without loss of activity of BDNF. The smaller protein also binds ATP and the corresponding charge state envelopes partially overlap those for BDNF. For annotations see Figure 1.

The methodology is currently used extensively for investigations with regard to complex formation in different systems changing the cation or the structure of the nucleotide and it has proven to be reliable. Its specificity was verified using ribonuclease $\mathrm{A}$ and lysozyme for controls. Ribonuclease A is known to bind ATP $[8,21]$. By applying our method we could demonstrate an ATP-ribonuclease A-complex. Lysozyme was used as a negative control. It has a $\mathrm{pI}$ in the basic range similar to that of GFs and there is no evidence for binding of ATP [22]. Accordingly, no complex formation between lysozyme and ATP could be observed using this methodology. Therefore, we can exclude that solely an ionic effect contributes to the interactions between the GFs and ATP.
The technique also allowed the time-dependent proteolytic digestion for further structure elucidation with the goal of localizing regions of the protein that are important for ATP binding. Complex decomposition turned out to be a fast reaction observable within only a few minutes. In the case of the globular structure of bFGF the results were of limited value; however, for NGF the loss of the N-terminal peptide SSSHPIFHR (amino acids 1-9) and the peptide EVMVLG EVNINNSVFK (amino acids 35-50; located at the top edge of the structure shown in Figure 1) did not seem to affect complex stability. The latter peptide is located the farthest away from the three disulfide bonds that determine the NGF structure and possibly also provide a stable groove for ATP binding. Currently, investigations are under way to elucidate the crystal structure of GF-ATP complexes.

\section{Conclusions}

The nature of the interaction of GF proteins and nucleotides was investigated. The formation of non-covalent ATP-GF complexes could be shown by the methodology introduced here that allows their selective purification and detection with MS. Main features of the procedure are: (1) reversed-phase purification of nucleotide-protein non-covalent complexes, (2) their detection with MALDI-TOF-MS using acid-free matrix, and/or (3) their measurement with ESI-MS. The method is not limited to the analysis of GFs, but might be expanded for the investigation of a wide range of proteins interacting with nucleotides as long as the complexes are sufficiently stable. The further characterization of their binding parameters is a prime task. Preliminary experiments suggest that one of the two presumed ATP-binding sites is located close to the three disulfide bridges in NGF.

\section{Acknowledgments}

We thank E. Schwarz (University of Halle, Germany) for the generous gift of the pET11a-proNGF plasmid and P. Madsen (University of Aarhus, Denmark) for providing the pET11aproBDNF plasmid. Figure 1 contains objects designed by ScienceSlides (ViviScience Corp., Chapel Hill, NC, USA), which are used with permission.

\section{References}

1. Klumpp, S.; Kriha, D.; Bechmann, G.; Maaßen, A.; Maier, S.; Pallast, S.; Hoell, P.; Krieglstein, J. Phosphorylation of the Growth Factors bFGF, NGF and BDNF: A Prerequisite for Their Biological Activity. Neurochem. Int. 2006, 48, 131-137.

2. Napper, S.; Kindrachuk, J.; Olson, D. J.; Ambrose, S. J.; Dereniwsky C.; Ross, A. R. Selective Extraction and Characterization of a HistidinePhosphorylated Peptide Using Immobilized Copper(II) Ion Affinity Chromatography and Matrix-Assisted Laser Desorption/Ionization Time-of-Flight Mass Spectrometry. Anal. Chem. 2003, 75, 1741-1747.

3. Medzihradszky, K. F.; Phillipps, N. J.; Senderowicz, L.; Wang, P.; Turck C. W. Synthesis and Characterization of Histidine-Phosphorylated Peptides. Protein Sci. 1997, 6, 1405-1411.

4. Wind, M.; Wegener, A.; Kellner, R.; Lehmann, W. D. Analysis of CheA Histidine Phosphorylation and Its Influence on Protein Stability by High-Resolution Element and Electrospray Mass Spectrometry. Anal. Chem. 2005, 77, 1957-1962. 
5. Nosworthy, N. J.; Peterkofsky, A.; König, S.; Seok, Y. J.; Szczepanowski, R. H.; Ginsburg, A. Phosphorylation Destabilizes the Amino-Terminal Domain of Enzyme I of the Escherichia coli Phosphenolpyruvate:Sugar Phosphotransferase System. Biochemistry 1998, 37, 6718-6726.

6. Collet, J. F.; Stoobant, V.; Van Schaftingen, E. Mechanistic Studies of Phosphoserine Phosphatase, an Enzyme Related to P-type ATPases. J. Biol. Chem. 1999, 274, 33985-33990.

7. Sobott, F.; McCammon, M. G.; Hernandez, H.; Robinson, C. V. The Flight of Macromolecular Complexes in a Mass Spectrometer. Philos. Trans. R. Soc. Lond. A 2005, 363, 379-391.

8. Camilleri, P.; Haskins, N. J. Investigating the Noncovalent Interaction of Cytidylic Acids with Ribonuclease A by Electrospray Mass-Spectrometry. Rapid Commun. Mass Spectrom. 1993, 7, 603-604

9. Pinkse, M. W. H.; Heck, A. J. R.; Rumpel, K.; Pullen, F. Probing Noncovalent Protein-Ligand Interactions of the cGMP-Dependent Protein Kinase Using Electrospray Ionization Time of Flight Mass Spectrometry. J. Am. Soc. Mass Spectrom. 2004, 15, 1392-1399.

10. Huang, E. C.; Pramanik, B. N.; Tsarbopoulos, A.; Reichert, P.; Ganguly, A. K.; Trotta, P. P.; Nagabhushan, T. L. Application of Electrospray Mass Spectrometry in Probing Protein-Protein and Protein-Ligand Noncovalent Interactions. J. Am. Soc. Mass Spectrom. 1993, 4, 624-630.

11. Ganguly, A. K.; Pramanik, B. N.; Tsarbopoulos, A.; Covey, T. R.; Huang, E.; Fuhrman, S. A. Mass-Spectrometric Detection of the Noncovalent Gdp-Bound Conformational State of the Human H-Ras Protein. J. Am. Chem. Soc. 1992, 114, 6559-6560.

12. König, S.; Hasche, A.; Pallast, S.; Krieglstein, J.; Klumpp, S. Proceedings of the 40th Annual Conference of the German Society of Mass Spectrometry, Bremen, March 11-14, 2007.

13. Rattenholl, A.; Lilie, H.; Grossmann, A.; Stern, A.; Schwarz, E.; Rudolph, R. The Pro-Sequence Facilitates Folding of Human Nerve Growth
Factor from Escherichia coli Inclusion Bodies. Eur. J. Biochem. 2001, 268, 3296-3303.

14. König, S.; Fales, H. M.; Haegele, K. D. Comment on the Cylindrical Capacitor Electrospray Interface. Anal. Chem. 1998, 70, 4453-4455.

15. Pitteri, S. J.; Chrisman, P. A.; Badman, E. R.; McLuckey, S. A. ChargeState Dependent Dissociation of a Trypsin/Inhibitor Complex via Ion Trap Collisional Activation. Int. J. Mass Spectrom. 2006, 253, 147-155.

16. Loboda, A. V.; Ackloo, S.; Chernushevich, I. V. A High-Performance Matrix-Assisted Laser Desorption/Ionization Orthogonal Time-ofFlight Mass Spectrometer with Collisional Cooling. Rapid Commun. Mass Spectrom. 2003, 17, 2508-2516.

17. Wang, Y.; Schubert, M.; Ingendoh, A.; Franzen, J. Analysis of NonCovalent Protein Complexes up to $290 \mathrm{kDa}$ Using Electrospray Ionization and Ion Trap Mass Spectrometry. Rapid Commun. Mass Spectrom. 2000, 14, 12-17.

18. Bothwell, M. A.; Shooter, E. M. Dissociation Equilibrium Constant of Beta Nerve Growth Factor. J. Biol. Chem. 1977, 252, 8532-8536.

19. Kastrup, J. S.; Eriksson, E. S.; Dalboge, H.; Flodgaard, H. X-ray Structure of the 154-Amino-acid Form of Recombinant Human Basic Fibroblast Growth Factor. Comparison with the Truncated 146-Amino-Acid Form. Acta Cryst. 1997, D53, 160-168.

20. McDonald, N. Q.; Lapatto, R.; Murray-Rust, J.; Gunning, J.; Wlodawer, A.; Blundell, T. L. New Protein Fold Revealed by a 2.3-A Resolution Crystal Structure of Nerve Growth Factor. Nature 1991, 354, 411-414.

21. Kumar, K.; Jenkins, J. L.; Jardine, A. M.; Shapiro, R. Inhibition of Mammalian Ribonuclases by Endogenous Adenosine Dinucleotides. Biochem. Biophys. Res. Commun. 2003, 300, 81-86.

22. Wetter, L. R.; Deutsch, H. F. Immunological Studies on Egg White Proteins. J. Biol. Chem. 1951, 192, 237-242. 